\title{
PREFERENCE DOMAINS AND THE MONOTONICITY OF CONDORCET EXTENSIONS ${ }^{\dagger}$
}

\author{
PAUL J. HEALY* AND MICHAEL PERESS**
}

\begin{abstract}
An alternative is a Condorcet winner if it beats all other alternatives in a pairwise majority vote. A social choice correspondence is a Condorcet extension if it selects the Condorcet winners-and nothing else-whenever a Condorcet winner exists. It is well known that Condorcet extensions are not monotonic (hence, not Nash implementable) when all preferences are admissible, but are implementable when restricted to a domain in which Condorcet winners always exist. We fill the gap by studying the intermediate domains, and find that monotonicity is violated on all such domains.
\end{abstract}

Keywords: Condorcet winner; Nash implementation; voting rules.

JEL Classification: D02, D71, D72.

\section{INTRODUCTION}

An alternative is a Condorcet winner if does not lose to any alternative in a pairwise majority vote. A social choice correspondence (SCC) is a Condorcet extension if it selects exactly the set of Condorcet winners, whenever a Condorcet winner exists. No restrictions are made when no Condorcet winner exists, except that the social choice correspondence must pick something at every preference profile. We say that we can (Nash) implement a Condorcet extension if there is a game form (or, mechanism) whose Nash equilibrium outcomes always exactly coincide with the SCC.

In this paper we study the preference domains on which we can implement Condorcet extensions. It is well known that Condorcet extensions are not Nash implementable when all strict or all weak preferences are admissible (Jackson, 2001; Saijo, 1987, e.g.). Maskin (1999) shows that Condorcet extensions are Nash implementable when only

\footnotetext{
${ }^{\dagger}$ The authors thank Yaron Azrieli for his helpful comments.

*Dept. of Economics, The Ohio State University, 1945 North High street, Columbus, Ohio 43210, U.S.A.; healy.52@osu.edu.

**Dept. of Political Science, Stony Brook University, Stony Brook, N.Y. 11794, U.S.A.; michael.peress@stonybrook.edu.
} 
preferences that admit Condorcet winners are admissible. ${ }^{1}$ Our paper explores the intermediate cases, where more preferences may be admissible than just those that admit Condorcet winners, but it is not assumed that all profiles are admissible. Our finding is that if the domain of admissible preference profiles is any strict superset of those profiles that admit Condorcet winners, then no Condorcet extension on that domain is Maskin monotonic. Therefore, it will be neither strategy-proof nor Nash implementable by any mechanism.

\section{Notation \& EnVironment}

A set of $N=\{1, \ldots, n\}$ of $n \geq 3$ agents are to select an outcome from a finite set of alternatives $X$, where $|X|$ (the number of elements of $X$ ) is at least two. Denote by $\mathscr{X}$ the set of non-empty subsets of $X$. Each agent has complete, reflexive, and transitive preferences $R_{i} \subseteq X \times X$, where $x R_{i} y$ denotes that $x$ is weakly preferred to $y$. Let $P_{i}$ denote the asymmetric part of $R_{i}$ ('strict preferences'). We denote the profile of all agents' preferences by $R_{N}=\left(R_{1}, \ldots, R_{n}\right)$. Let $\mathscr{R}$ be the space of all possible preferences over $X$ and $\mathscr{P}$ be the space of all strict preferences over $X$.

Given a set of admissible preference profiles $\mathscr{D} \subseteq \mathscr{R}^{n}$, a social choice correspondence (SCC) is a mapping $f: \mathscr{D} \rightarrow \mathscr{X}$ that selects a set of alternatives for each profile $R_{N}$. Note that, by definition, $f\left(R_{N}\right) \neq \varnothing$ for all $R_{N} \in \mathscr{D}$. If $f$ is single valued, we refer to it as a social choice function (SCF). For any subset $\mathscr{A} \subseteq \mathscr{D}$, let $\left.f\right|_{\mathscr{A}}$ be the restriction of $f$ to profiles in $\mathscr{A}$.

Define $N\left(x, y ; R_{N}\right)=\left|\left\{i \in N: x P_{i} y\right\}\right|$ to be the number of agents who strictly prefer $x$ over $y$ at profile $R_{N}$. An alternative $x$ is said to be a weak Condorcet winner at $R_{N}$ if, for every $y \in X \backslash\{x\}, N\left(x, y ; R_{N}\right) \geq N\left(y, x ; R_{N}\right)$. In other words, a weak Condorcet winner $x$ does not lose a pairwise plurality vote against any other alternative, assuming indifferent voters abstain. There are many preference profiles for which no weak Condorcet winner exists. Let $\mathbb{W}=\left\{R_{N} \in \mathscr{R}^{n}:(\exists x \in X)(\forall y \in X), N\left(x, y ; R_{N}\right) \geq N\left(y, x ; R_{N}\right)\right\}$ be the set of preference profiles that admit a weak Condorcet winner. Define $f^{W}: \mathscr{W} \rightarrow \mathscr{X}$ to be the SCC that selects all weak Condorcet winners for any $R_{N} \in \mathbb{W}$. A SCC $f$ is said to be a weak Condorcet extension if $f\left(R_{N}\right)=f^{W}\left(R_{N}\right)$ whenever $R_{N} \in \mathbb{W}$, and weak Condorcet consistent if $f\left(R_{N}\right) \subseteq f^{W}\left(R_{N}\right)$ whenever $R_{N} \in \mathbb{W}$. No restrictions are placed on weak Condorcet extensions or weak Condorcet consistent SCCs outside of $\mathbb{W}$.

There can be multiple weak Condorcet winners at a given preference profile. For example, if all agents are indifferent over all alternatives, then all alternatives are weak

\footnotetext{
${ }^{1}$ The domain restriction in Maskin's paper is not discussed explicitly, but clear from the proof.
} 
Condorcet winners. Define $x \in X$ to be a strong Condorcet winner at $R_{N}$ if, for every $y \in X \backslash\{x\}, N\left(x, y ; R_{N}\right)>N\left(y, x ; R_{N}\right)$. Strong Condorcet winners must be unique when they exist. Let $\mathscr{S} \subseteq \mathscr{R}^{n}$ be the preference profiles for which a strong Condorcet winner exists, and $f^{S}: \mathscr{S} \rightarrow X$ to be the SCF that selects the strong Condorcet winner at every $R_{N} \in \mathscr{S}$. A SCC $f$ is a strong Condorcet extension (or, equivalently, strong Condorcet consistent) if $f\left(R_{N}\right)=f^{S}\left(R_{N}\right)$ whenever $R_{N} \in \mathscr{S}$. Obviously, $\mathscr{S} \subset W^{2}$ Furthermore, every weak Condorcet extension is a weak Condorcet consistent SCC, and every weak Condorcet consistent SCC is a strong Condorcet extension, but the opposite relations do not hold.

To define monotonicity, we first say that an alternative $x \in X$ maintains position from $R_{N}$ to $R_{N}^{\prime}$ if, for every $i \in N, x R_{i} y$ implies $x R_{i}^{\prime} y$. In other words, $x$ maintains position if, for every $i$, everything $x$ was beating under $R_{i}$ continues to be beaten by $x$ under $R_{i}^{\prime}$. A SCC $f$ is monotonic if, whenever $x \in f\left(R_{N}\right)$ and $x$ maintains position from $R_{N}$ to $R_{N}^{\prime}$, then $x \in f\left(R_{N}^{\prime}\right)$.

A mechanism $\Gamma=(S, g)$ consists of a strategy space $S=\times{ }_{i=1}^{n} S_{i}$ and an outcome function $g: S \rightarrow X$. A strategy profile $s^{*}$ is a (pure strategy) Nash equilibrium of $\Gamma$ at $R_{N}$ if, for every $i \in N$ and $s_{i} \in S_{i}, g\left(s^{*}\right) R_{i} g\left(s_{i}, s_{-i}^{*}\right)$. For any $R_{N}$, let $\mu_{\Gamma}\left(R_{N}\right)$ identify the set of pure-strategy Nash equilibria of $\Gamma$ at $R_{N}$. The mechanism $\Gamma$ Nash implements a SCC $f$ if, for every $R_{N}, g\left(\mu_{\Gamma}\left(R_{N}\right)\right)=f\left(R_{N}\right)$. In that case we say that $f$ is Nash implementable.

The following theorem, due to Maskin (1999), shows that monotonicity is an important necessary condition for a SCC to be Nash implementable.

Theorem (Maskin, 1999). If a SCC $f: \mathscr{D} \rightarrow \mathscr{X}$ is not monotonic, then it is not Nash implementable.

Maskin (1999) also proves that, with at least three agents, monotonicity is sufficient for Nash implementation when the 'No Veto Power' axiom is added. Formally, $f$ satisfies No Veto Power if $\left|\left\{i \in N: x R_{i} y \forall y \in X\right\}\right| \geq n-1$ implies $x \in f\left(R_{N}\right)$.

Theorem (Maskin, 1999). If $n \geq 3$ and $f: \mathscr{D} \rightarrow \mathscr{X}$ satisfies monotonicity and No Veto Power then $f$ is Nash implementable.

We state and prove our result in the following section.

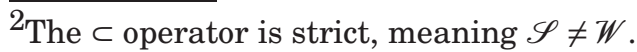




\section{The Main Result}

Theorem. If $\mathscr{D} \subseteq \mathscr{S} \subset \mathbb{W}$, then

(1) all strong Condorcet extensions are monotonic and Nash implementable,

(2) all weak Condorcet consistent SCCs are monotonic and Nash implementable, and

(3) all weak Condorcet extensions are monotonic and Nash implementable. If $\mathscr{D} \subseteq \mathscr{W}$, then

(4) strong Condorcet extensions may or may not be monotonic,

(5) weak Condorcet consistent SCCs may or may not be monotonic, and

(6) all weak Condorcet extensions are monotonic and Nash implementable. If $\mathscr{S} \subset \mathbb{W} \subset \mathscr{D}$, then

(7) no strong Condorcet extension is monotonic,

(8) no weak Condorcet consistent SCC is monotonic, and

(9) no weak Condorcet extension is monotonic.

Maskin (1999) essentially proves results $1-3$ and 6. The novelty of our theorem is in the impossibility of Nash implementation when $\mathbb{W} \subset \mathscr{D}$ (results 7-9). The argument is simple: Following result 9, suppose $f$ is a weak Condorcet extension. Take any $R_{N} \notin \mathbb{W}$, where no weak Condorcet winner exists (e.g., panel A of Table I). For every $x \in f\left(R_{N}\right)$, there is some $y$ that strictly beats $x$ in a pairwise majority vote at $R_{N}$. Now consider the profile $R_{N}^{\{x, y\}}$ that is identical to $R_{N}$, except $x$ and $y$ are 'floated' to the top of each agent's preference ranking (panel B of Table I). Here, $y$ is the unique (strong) Condorcet winner. This means $R_{N}^{\{x, y\}} \in \mathscr{S} \subset \mathscr{D}$, so $f$ is defined at $R_{N}^{\{x, y\}}$. In fact, we know $f\left(R_{N}^{\{x, y\}}\right)=\{y\}$ since $f$ is a weak Condorcet extension. Now, can $f$ be monotonic? By construction, $x$ (which is in $\left.f\left(R_{N}\right)\right)$ maintains position from $R_{N}$ to $R_{N}^{\{x, y\}}$, so monotonicity would require $x \in f\left(R_{N}^{\{x, y\}}\right)$. But we've just shown that $f\left(R_{N}^{\{x, y\}}\right)=\{y\}$, so $f$ cannot be monotonic. A similar example proves results 7 and 8. Arguments such as this are common in past work; see Amorós (2009), for example.

Details are provided in the following proof.

Proof of the Theorem. For results $1-3$, consider $\left.f \equiv f^{S}\right|_{\mathscr{D}}$, which is the unique strong Condorcet extension, the unique weak Condorcet consistent SCC, and the unique weak Condorcet extension on $\mathscr{D}$. Pick any $R_{N}, R_{N}^{\prime} \in \mathscr{D} \subseteq \mathscr{S}$. If the element $x$ such that $f\left(R_{N}\right)=$ $\{x\}$ maintains position from $R_{N}$ to $R_{N}^{\prime}$, then $N\left(x, y ; R_{N}^{\prime}\right) \geq N\left(x, y ; R_{N}\right) \geq N\left(y, x ; R_{N}\right) \geq$ $N\left(y, x ; R_{N}^{\prime}\right)$ for all $y$. Thus, $f\left(R_{N}^{\prime}\right)=\{x\}$, as required by monotonicity. This SCC also 


$\begin{array}{cccccc}\frac{R_{1}}{z} & \frac{R_{2}}{x} & \frac{R_{3}}{y} & \frac{R_{1}^{\{x, y\}}}{y} & \frac{R_{2}^{\{x, y\}}}{x} & \frac{R_{3}^{\{x, y\}}}{y} \\ y & z & x & x & y & x \\ x & y & z & z & z & z\end{array}$

TABLE I. Example preferences illustrating the proof.

satisfies No Veto Power since $n-1$ agents ranking $x$ as top-ranked at $R_{N}$ guarantees that $x$ is the strong Condorcet winner at $R_{N}$, and therefore $f\left(R_{N}\right)=\{x\}$. Thus, $f$ is Nash implementable.

For results 4 and 5, consider an example in which $X=\{x, y\}$ and $\mathscr{D}=\mathscr{S} \cup\left\{R_{N}^{\prime}, R_{N}^{\prime \prime}\right\}$, where $R_{N}^{\prime} \in \mathbb{W}$ is any profile such that $N\left(x, y ; R_{N}^{\prime}\right)=N\left(y, x ; R_{N}^{\prime}\right)$ and $R_{N}^{\prime \prime} \in \mathbb{W}$ is the profile where all agents are indifferent between $x$ and $y$. Note that $f^{W}\left(R_{N}^{\prime}\right)=f^{W}\left(R_{N}^{\prime \prime}\right)=\{x, y\}$. Suppose $\left.f\right|_{\mathscr{S}} \equiv f^{S}, f\left(R_{N}^{\prime}\right)=\{x\}$, and $f\left(R_{N}^{\prime \prime}\right)=\{y\}$. Then $f$ is a strong Condorcet extension and a weak Condorcet consistent SCC, but is not a weak Condorcet extension. It is also not monotonic: $x$ is chosen at $R_{N}^{\prime}$ and $x$ maintains position from $R_{N}^{\prime}$ to $R_{N}^{\prime \prime}$, but $x \notin f\left(R_{N}^{\prime \prime}\right)$. Thus, monotonicity fails for some strong Condorcet extensions and some weak Condorcet consistent SCCs. To show there exists monotonic strong Condorcet extensions and weak Condorcet consistent SCCs, let $\left.f \equiv f^{W}\right|_{\mathscr{D}}$. Here, if $x \in f\left(R_{N}\right)$ for some $R_{N} \in \mathscr{D} \subseteq \mathbb{W}$ then $x$ is a weak Condorcet winner at $R_{N}$. If $x$ maintains position from $R_{N}$ to $R_{N}^{\prime} \in \mathscr{D}$, then $N\left(x, y ; R_{N}^{\prime}\right) \geq N\left(x, y ; R_{N}\right) \geq N\left(y, x ; R_{N}\right) \geq N\left(y, x ; R_{N}^{\prime}\right)$ for all $y$. Thus, $x$ is a weak Condorcet winner at $R_{N}^{\prime}$ and so $x \in f\left(R_{N}^{\prime}\right)$, as required by monotonicity. All weak Condorcet extensions on such a domain must satisfy $\left.f \equiv f^{W}\right|_{\mathscr{D}}$, so this also proves result 6 .

To show results $7-9$, recall that assume $\mathbb{W} \subset \mathscr{D}$. Pick any $R_{N} \in \mathscr{D} \backslash \mathscr{W}$ and any $x \in$ $f\left(R_{N}\right)$. Since there is no weak Condorcet winner at $R_{N}$, then there is some $y \in X$ such that $N\left(y, x ; R_{N}\right)>N\left(x, y ; R_{N}\right)$. Now consider the preference relation $R_{N}^{\{x, y\}}$ where, for each $i$,

(1) $x R_{i} y \Rightarrow x R_{i}^{\{x, y\}} y P_{i}^{\{x, y\}} z$ for every $z \notin\{x, y\}$,

(2) $y R_{i} x \Rightarrow y R_{i}^{\{x, y\}} x P_{i}^{\{x, y\}} z$ for every $z \notin\{x, y\}$, and

(3) $w R_{i} z \Rightarrow w R_{i}^{\{x, y\}} z$ for all $w, z \notin\{x, y\}$.

In other words, $R_{i}^{\{x, y\}}$ is identical to $R_{i}$ except the pair $\{x, y\}$ is 'bubbled up' to the top of $R_{i}$. Note that $N\left(y, x ; R_{N}^{\{x, y\}}\right)>N\left(x, y ; R_{N}^{\{x, y\}}\right)$ since $N\left(y, x ; R_{N}\right)>N\left(x, y ; R_{N}\right)$, and also $n=N\left(y, z ; R_{N}\right)>N\left(z, y ; R_{N}\right)=0$ for all $z \notin\{x, y\}$. Thus, the unique weak Condorcet winner at $R_{N}^{\{x, y\}}$ is $y$, which also verifies that $R_{N}^{\{x, y\}} \in \mathscr{S} \subseteq \mathscr{D}$. If $f$ is a weak Condorcet 
extension, weak Condorcet consistent, or a strong Condorcet extension, it must be that $f\left(R_{N}^{\{x, y\}}\right)=\{y\}$.

Now we ask whether $f$ can be monotonic. By construction, $x \in f\left(R_{N}\right)$ maintains position from $R_{N}$ to $R_{N}^{\{x, y\}}$, so monotonicity would require that $x \in f\left(R_{N}^{\{x, y\}}\right)$. But we have just derived that $f\left(R_{N}^{\{x, y\}}\right)=\{y\}$, a contradiction, so $f$ cannot be monotonic.

Remark 1. If $\mathscr{D}$ does not contain all of $\mathscr{W}$ but does contain some profiles outside of $\mathscr{W}$ then the impossibility result remains true as proven as long as, for every $R_{N} \in \mathscr{D} \backslash \mathbb{W}$, there is some $R_{N}^{\prime}$ that plays the same role as $R_{N}^{\{x, y\}}$ in the proof.

Remark 2. We assume $n \geq 3$. If $n=2$ then every preference profile admits a weak Condorcet winner $\left(\mathbb{W}=\mathscr{R}^{2}\right)$, but there are weak Condorcet extensions that are not Nash implementable when $\mathscr{D}$ is large enough. Examples can be constructed easily using the necessary conditions from Dutta and Sen (1991).

\section{RELATED WORK}

As stated in the introduction, the impossibility result under the special cases of $\mathscr{D}=\mathscr{P}^{n}$ and $\mathscr{D}=\mathscr{R}^{n}$ is well-studied. Assuming $|X| \geq 3$ and $\mathscr{D} \supseteq \mathscr{P}^{n}$, (all strict preferences are admissible), Muller and Satterthwaite (1977) prove that if $f$ is monotonic and satisfies citizens' sovereignty (the range of $f$ equals $X$ ) then $f$ must be dictatorial. Since a weak Condorcet extension on $\mathscr{P}^{n}$ satisfies citizen sovereignty and is not dictatorial, this proves that it cannot be monotonic.

Amorós (2009) defines the unequivocal majority of a social choice function to be the minimal number of agents such that if that many agents rank an alternative $x$ at the top of the preferences, then the social choice function picks $x$. For weak Condorcet extensions the unequivocal majority is $n / 2$. Assuming all strict preferences are admissible $\left(\mathscr{D}=\mathscr{P}^{n}\right)$, Amorós shows that if the unequivocal majority of a social choice correspondence is less than $n-(n-1) / m$ (where $m$ is the number of alternatives) then it cannot be Maskin monotonic. Since $n / 2+1<n-(n-1) / m$, this also shows that weak Condorcet extensions are not monotonic when all strict preferences are admissible.

Ozkal-Sanver and Sanver (2007) also show the impossibility of implementing weak Condorcet extensions when all preferences are admissible. Their approach differs in that they take the (non-transitive) majority relation as primitive, but the result is equivalent. 
All of these results require that all strict preferences be admissible, and are therefore more restrictive (more likely to generate an impossibility result) than any of the cases covered in our theorem.

If the complete indifference profile is admissible, Saijo (1987) shows that Maskin monotonicity of a (single-valued) SCF implies that the SCF is constant. Thus, if $\mathscr{D}$ contains the indifference profile and two other profiles in $\mathbb{W}$ that have non-overlapping sets of weak Condorcet winners, then any weak Condorcet consistent SCF defined on $\mathscr{D}$ would be non-constant and therefore not monotonic. Such a domain could be a subset of $\mathbb{W}$, in which case Saijo's result is a special case of (5) above. Or it could be that $\mathscr{D}$ is neither a superset nor a subset of $\mathbb{W}$, in which case Saijo's impossibility result is not covered by our theorem.

Positive results on implementation can be obtained if a stronger equilibrium concept is used (see Palfrey and Srivastava, 1991; Peress, 2008; and Bag et al., 2009, for example). If preferences are single-peaked (Moulin, 1980, e.g.) then implementation is also no problem, because $\mathscr{D} \subset \mathscr{S}$.

\section{REFERENCES}

Amorós, P., 2009. Unequivocal majority and maskin monotonicity. Social Choice and Welfare 33, 521-532.

Bag, P. K., Sabourian, H., Winter, E., 2009. Multi-stage voting, sequential elimination and condorcet consistency. Journal of Economic Theory 124, 1278-1299.

Dutta, B., Sen, A., 1991. A necessary and sufficient condition for two-person nash implementation. Review of Economic Studies 58, 121-128.

Jackson, M. O., 2001. A crash course in implementation theory. Social Choice and Welfare 18, 655-708.

Maskin, E., 1999. Nash equilibrium and welfare optimality. Review of Economic Studies $66,23-38$.

Moulin, H., 1980. On strategy-proofness and single-peakedness. Public Choice 35, 437455.

Muller, E., Satterthwaite, M. A., 1977. The equivalence of strong positive association and strategy-proofness. Journal of Economic Theory 14, 412-418.

Ozkal-Sanver, I., Sanver, M. R., February 2007. Nash implementability of tournament solutions, Istanbul Bilgi University Working Paper.

Palfrey, T. R., Srivastava, S., 1991. Nash implementation using undominated strategies. Econometrica 59, 479-501. 
Peress, M., 2008. Selecting the condorcet winner: Single-stage versus multi-stage voting rules. Public Choice 137, 207-222.

Saijo, T., 1987. On constant maskin monotonic social choice functions. Journal of Economic Theory 42 (2), 382-386. 